\title{
Urinary tract infections in pregnancy: old and new unresolved diagnostic and therapeutic problems
}

Joanna Matuszkiewicz-Rowińska1 ${ }^{1}$ Jolanta Małyszko², Monika Wieliczko ${ }^{1}$

\begin{abstract}
${ }^{1}$ Chair and Clinic of Nephrology, Dialysis and Internal Diseases, Medical University of Warsaw, Warsaw, Poland

${ }^{2}$ Department of Nephrology and Transplantation, Medical University of Bialystok, Bialystok, Poland
\end{abstract}

Submitted: 10 June 2013

Accepted: 23 September 2013

Arch Med Sci 2015; 11, 1: 67-77

DOI: 10.5114 /aoms.2013.39202

Copyright (c) 2015 Termedia \& Banach

\section{Abstract}

Urinary tract infections (UTIS) are common in pregnant women and pose a great therapeutic challenge, since the risk of serious complications in both the mother and her child is high. Pregnancy is a state associated with physiological, structural and functional urinary tract changes which promote ascending infections from the urethra. Unlike the general population, all pregnant women should be screened for bacteriuria with urine culture, and asymptomatic bacteriuria must be treated in every case that is diagnosed, as it is an important risk factor for pyelonephritis in this population. The antibiotic chosen should have a good maternal and fetal safety profile. In this paper, current principles of diagnosis and management of UTI in pregnancy are reviewed, and the main problems and controversies are identified and discussed.

Key words: pregnancy, asymptomatic bacteriuria, acute cystitis, acute pyelonephritis.

\section{Introduction}

Urinary tract infections (UTIs) in pregnant women continue to pose a clinical problem and a great challenge for physicians. Although the incidence of bacteriuria in this population is only slightly higher than in non-pregnant women, its consequences for both the mother and the unborn child are more severe. There is a much higher risk (up to 40\%) of progression to pyelonephritis, and possibly increased risk of pre-eclampsia, premature birth and low neonatal birth weight [1-6]. That is related to profound structural and functional urinary tract changes, typical for pregnancy. In about $80 \%$ of pregnant women dilation of the urinary tract combined with slight hydronephrosis is observed, caused partly by a reduction in smooth muscle tone with slowing of ureteral peristalsis, and partly by urethral sphincter relaxation. This may be due to high levels of circulating progesterone $[1,7]$. Simultaneously, the enlarged uterus compresses the urinary bladder, thus increasing the intravesical pressure, which may result in vesico-ureteral reflux and urine retention in the bladder after miction, commonly observed in pregnant women. Urinary stasis and impairment of the physiological anti-reflux mechanism create conditions favorable for bacterial growth and ascending infection.
Corresponding author:

Prof. Joanna MatuszkiewiczRowińska MD, PhD

Chair and Clinic

of Nephrology, Dialysis and Internal Diseases Medical University of Warsaw Independent Public Central Clinical Hospital

1 a Banacha St 02-097 Warsaw, Poland Phone: +48 2259913 14, +48225992658

Fax: +48 225991658

E-mail: jrowinska@gmail.com 
The additional predisposing factors include pregnancy-specific biochemical changes in urine, with higher amounts of glucose, amino acids and hormone degradation products, which increase urinary $\mathrm{pH}[7,8]$.

Similarly as in non-pregnant women, in pregnant women UTIs are classified either as asymptomatic bacteriuria (ASB), when the infection is limited to bacterial growth in urine, or symptomatic infections (acute cystitis, acute pyelonephritis), when bacteria invade urinary tract tissues, inducing an inflammatory response. The UTIs in pregnancy are by definition considered complicated infections and require a special diagnostic approach and management.

\section{Epidemiology and risk factors}

Urinary tract infections remain among the most common medical complications during pregnancy. It is estimated that the prevalence of ASB varies between $2 \%$ and $10-13 \%$, similar to nonpregnant women [9-13]. There is a scarcity of data concerning acute cystitis in pregnancy; according to the available studies it is observed in $1-4 \%[11,14,15]$ The prevalence of acute pyelonephritis in most reports ranges from $0.5 \%$ to $2 \%$ of pregnancies [ 1 , $8,16,17]$.

Many women acquire bacteriuria before pregnancy $[18,19]$. A large retrospective analysis with logistic regression modeling, embracing 8037 women from North Carolina, revealed that the two strongest predictors of bacteriuria at prenatal care at prenatal care initiation were: UTI prior to prenatal care initiation $(\mathrm{OR}=2.5,95 \% \mathrm{Cl}$ : 0.6-9.8 for whites, and $\mathrm{OR}=8.8,95 \% \mathrm{Cl}: 3.8-20.3$ for blacks) and a pre-pregnancy history of UTI (OR $=2.1,95 \% \mathrm{Cl}: 1.4-3.2$ ) [19]. In a second analysis, prior antenatal UTI was found to be the strongest predictor of pyelonephritis after 20 weeks' gestation $(\mathrm{OR}=5.3,95 \% \mathrm{Cl}: 2.6-11.0)$ [20]. Other suggested risk factors for UTI during pregnancy are lower socioeconomic status, sexual activity, older age, multiparity, anatomical urinary tract abnormalities, sickle cell disease and diabetes, although the significance of some of them (age, parity or sickle cell trait) remains a matter of controversy [1, 10, 21-23].

\section{Microbiology}

The pathogens responsible for infections during pregnancy are similar to those in the general population. Most infections are caused by Enterobacteriaceae, commonly found in the gastrointestinal tract, with Escherichia coli responsible for 63-85\% of cases, and among the remaining: Klebsiella pneumoniae ( $8 \%)$, coagulase-negative Staphylococcus (up to $15 \%$ ), S. aureus (up to $8 \%$ ), and group B streptococci (GBS) (2-7\%) [16, 17, 24-26].

\section{Consequences of urinary tract infection in pregnancy}

\section{Asymptomatic bacteriuria}

The only serious maternal consequence of untreated ASB in pregnant women is a significant risk of acute pyelonephritis in later pregnancy (30-40\% vs. $3-4 \%$ in treated patients) [27].

The results of the studies on perinatal outcomes of untreated ASB are controversial. Although a number of them demonstrated a relationship of ASB in pregnant mothers and the risk of premature delivery and/or lower birth weight, some other studies failed to prove the association [28-32]. The Cochrane Library meta-analysis revealed that antibiotic treatment was effective in reducing the incidence of low-birth-weight infants but not of preterm deliveries [27]. However, the authors stressed the poor methodological quality of the available studies, their different design, lack of sufficient information about the randomization methods, different definitions used, low statistical power and some substantial biases, urging caution in drawing conclusions. A good example of these problems is presented by the Cardiff Birth Survey [33]. In a prospectively studied large cohort of 25844 pregnancies, several demographic, social and medical factors (including bacteriuria) were significantly associated with preterm birth in the initial univariable analyses. However, after adjustments for other medical factors, bacteriuria retained an association of only borderline significance, and after further adjustment for demographic and social factors, the relationship completely disappeared. The results of the second analysis of the same cohort, aimed to compare associations of studied factors with spontaneous vs. indicated preterm birth, are even more interesting [34]. Two separate multiple logistic regression analyses revealed that spontaneous and indicated preterm births have different overall profiles of risk factors, and only the last of them was associated with bacteriuria. The authors concluded that ASB, if it does not progress to symptomatic UTI, is not associated with preterm delivery.

Maternal GBS bacteriuria in a pregnant woman is considered a marker for genital tract colonization with this organism which poses a significant risk of preterm rupture of the membranes, premature delivery and early-onset severe neonatal infection [1, 24, 26, 35-37].

\section{Symptomatic urinary tract infection}

About $15-20 \%$ of women with pyelonephritis have bacteremia $[8,17]$. They may develop various complications, such as acute kidney injury, anemia, hypertension, preeclampsia, sepsis and septic shock, hemolysis, thrombocytopenia, and acute respiratory distress syndrome, particularly if treat- 
ment is initiated too late [17, 27, 38-44]. Although these associations have not always been proved to be causal, most of the complications seem to be due to renal or other tissue damage caused by bacterial endotoxins and a systemic inflammatory response with endothelial injury $[42,45]$.

A number of observational studies have demonstrated the relationship between maternal symptomatic UTI and the risk of premature delivery and lower birth weight $[28-30,46]$. The frequency of preterm deliveries in women with acute pyelonephritis is significantly higher than in women free of this complication, and pyelonephritis seems to be an important independent risk factor for delivery before 37 weeks' gestation [2, 5, 47]. However, again, a substantial heterogeneity between these studies, together with many possible biases, makes it difficult to establish the overall contribution of UTI to preterm birth [48]. A rare but severe complication is the transmission of the infection onto the newborn baby [49]. Very often the transmitted infection originates from a heavily colonized birth canal, usually with GBS [26].

\section{Safety of antimicrobial treatment}

Nearly all antimicrobials cross the placenta, and some of them may exert teratogenic effects. Commonly accepted antibiotics used in treating UTIs during pregnancy, regardless of its period, include derivatives of penicillin and cephalosporins, particularly those with low protein-binding ability (such as cephalexin), all of FDA pregnancy category B (Table I) [50].

Nitrofurantoin and trimethoprim/sulfamethoxazole should be avoided during the first trimester due to a possible risk of fetal defects, although the studies on that issue yield somewhat contradictory results [1, 51-53]. In the large American population-based National Birth Defects Prevention Study, maternal use of sulfonamides and nitrofurantoin (1 month before pregnancy to the end of the first trimester) was associated with more serious defects than any other antibacterial classes [51]. However, this study has been criticized for several significant limitations including recall bias (women were asked about antibiotic use after pregnancy and it was not confirmed by medical records), inability to determine whether the birth defect was due to the antibiotic itself, the infection for which the antibiotic was prescribed, or other confounding factors. Two years later, the Committee of Obstetrics Practice of the American College of Obstetricians and Gynecologists, summarizing the available data on the relationship between prenatal exposure to both antimicrobials and birth defects, concluded that: 1) "When selecting an antibiotic for a true infection during the first trimester of pregnancy (that is, during organogenesis), health care providers should consider and discuss with patients the benefits as well as the potential unknown risks of teratogenesis and maternal adverse reactions; 2) "Prescribing sulfonamides or nitrofurantoin in the first trimester is still considered appropriate when no other suitable alternative antibiotics are available"; 3) "Pregnant women should not be denied appropriate treatment for infections because untreated infections can commonly lead to serious maternal and fetal complications" [52]. Recently Nordeng et al. published the results from a large

Table I. US Food and Drug Administration (FDA) categories of medications in pregnancy

\begin{tabular}{|c|c|c|c|}
\hline Antibiotic & FDA risk category & Antibiotic & FDA risk category \\
\hline Amoxicillin & B & Trimethoprim/sulfamethoxazol & C \\
\hline Cephalosporins & B & Ciprofloxacin & C \\
\hline Piperacillin/tazobactam & B & Levofloxacin & C \\
\hline Daptomycin & B & Imipenem/cilastatin & C \\
\hline Azithromycin & B & Linezolid & $\mathrm{C}$ \\
\hline Erythromycin & B & Clarithromycin & $\mathrm{C}$ \\
\hline Meropenem & B & Spiramycin & C \\
\hline Clindamycin & B & Gentamycin & C \\
\hline Nitrofurantoin & B & Amikacin & $\mathrm{D}$ \\
\hline Vankomycin iv. & B & Tobramycin & $\mathrm{D}$ \\
\hline Metronidazol iv. & B & Netilmycin & D \\
\hline Trimethoprim & C & Tetracyclines & $\mathrm{D}$ \\
\hline
\end{tabular}

A - Well-controlled studies available in humans with no adverse effects observed in human pregnancies; $B$ - No adverse effects in wellcontrolled studies of human pregnancies with adverse effects seen in animal pregnancies $O R$ no adverse effects in animal pregnancies without well-controlled human pregnancy data available; C - Human data lacking with adverse pregnancy effects seen in animal studies OR no pregnancy data available in either animals or humans; $D$ - Adverse effects demonstrated in human pregnancies; benefits of drug use may outweigh the associated risks. 
population-based cohort study using the Norwegian Prescription Database linked to data on all live births, stillbirths, and induced abortions after 12 weeks of gestation from the Medical Birth Registry of Norway [53]. Among 180120 pregnancies between 2004 and 2008, 1334 women filled prescriptions for nitrofurantoin in the first trimester. The authors found that dispensing nitrofurantoin during the first trimester was not associated with increased risk of major malformations $(O R=0.79$, $95 \% \mathrm{Cl}: 0.51-1.23)$ or higher rates of stillbirth, neonatal death, low birth weight, or preterm delivery.

In the second and third trimester, trimethoprim/sulfamethoxazole and nitrofurantoin are well tolerated and by some considered even first line agents, except in the last week before delivery, when they may increase neonatal jaundice and predispose to kernicterus [1, 10, 51-55]. The same concerns other antimicrobials with very high protein binding (e.g. ceftriaxone), since they can displace bilirubin from plasma proteins. One should also remember that trimethoprim (FDA pregnancy category C) is a folic acid antagonist; thus, supplementation of this agent and monitoring of its serum concentration are required during treatment $[56,57]$. Nitrofurantoin can be theoretically associated with a risk of fetal or neonatal hemolytic anemia if the mother has glucose-6-phosphate deficiency, and although this complication in pregnancy has not been reported, the drug should be used with caution, particularly in areas of disease prevalence $[10,58,59]$.

The use of fluoroquinolones (FDA pregnancy category C) is essentially contraindicated throughout pregnancy, since fetal cartilage development disorders have been reported in experimental animals, although not in human studies [27, 60-63]. In the largest study so far, 200 pregnant women exposed to fluoroquinolones were compared to 200 women exposed to nonteratogenic, nonembryotoxic antimicrobials, matched by indication, duration of therapy ( $\sim 3$ days), and trimester of exposure [60]. The rate of major congenital malformations did not differ between the group exposed to quinolones in the first trimester and the control group $(2.2 \%$ vs. $2.6 \%$; RR $=0.85 ; 95 \% \mathrm{Cl}$ : 0.21-3.49) and was within the expected normal range (1-5\%). A systemic review of prospective, controlled studies showed that the use of fluoroquinolones during the first trimester of pregnancy does not appear to be associated with an increased risk of major malformations recognized after birth, stillbirths, preterm births or low birth weight [64]. Apparently more data are needed to establish safety of fluoroquinolones in pregnancy before they may be routinely prescribed. However, in some cases of complicated symptomatic UTI, resistant to other antibiotics, their benefits may outweigh the risks [60].
Gentamicin and other aminoglycosides are FDA pregnancy category $D$, because of their potential nephro- and neurotoxicity (eighth nerve damage) to the fetus $[1,65,66]$. Tetracyclines lead to discoloration of deciduous teeth if given after 5 months' gestation [65, 66]. Macrolides have been assigned to pregnancy category $C$ by the FDA (Table I). Although these drugs are used in pregnancy relatively often, the data on their embryotoxicity and teratogenicity are limited. Earlier reports suggested an association between prenatal exposure to macrolides and congenital heart defects or pyloric stenosis, whereas the results of some recent studies are rather reassuring [51, 6771]. The first prospective controlled multicenter study of exposure to clarithromycin in early pregnancy suggested that this agent does not increase the risk of fetal malformations above the expected $1-3 \%$ [68]. However, there was a two-fold higher rate (14\% vs. $7 \%)$ of spontaneous abortions in the exposed vs. control group, and although it still remained within the expected baseline rate, the possibility that it could be a result of undiagnosed fetal malformation cannot be excluded. No significant teratogenic effect of erythromycin was identified in a Hungarian case-control study, a nationally based registry of cases with congenital abnormalities [69]. The main limitations of this data set were: a relatively low response rate, retrospective collection of data (recall bias), inability to exclude the effect of other drugs, and a restriction of the study to the second and third trimester. However, in a large prospective observational study, performed in 511 women exposed to macrolides during the first trimester, Bar-Oz et al. did not observe a significant difference in the rate of major congenital malformations between the study group and comparison group [70]. Recently, Lin et al. compared the prenatal usage of erythromycin and nonerythromycin macrolides by mothers of 4132 infants with a congenital heart defect and 735 with pyloric stenosis and mothers of 6952 infants without any malformation, serving as a control group [71]. In logistic regression analysis they found no association of exposure to the drugs and increased risk of both types of birth defects. Again, these results should be interpreted with caution, since the power of the study was limited and - as the authors underline - modest associations could be missed. So further studies are needed before the macrolides become accepted for wide use. Until then, this group of antibiotics should be reserved for the treatment of serious or life-threatening conditions, unresponsive to standard antibiotic therapy.

Interesting findings came from the ORACLE Children Study II, which assessed the long-term outcomes for 3190 children born to women who had received antibiotics vs. placebo for threatened 
preterm labor with intact membranes [72]. The study sought follow-up information for children at age 7 in the UK using a parent-report postal questionnaire. The authors found that exposure to erythromycin or amoxicillin-clavulanate significantly increased the number of children with various functional impairments and cerebral palsy compared to placebo $(\mathrm{OR}=1.42,95 \% \mathrm{Cl}$ : $0.68-$ 2.98 , and $\mathrm{OR}=1.22,95 \% \mathrm{Cl}: 0.57-2.62$, respectively). The risk was greatest when both antibiotics were given together compared to double placebo $(\mathrm{OR}=2.91,95 \% \mathrm{Cl}: 1.50-5.65)$. The cause of this neurological dysfunction is unclear, but it could be a result of subclinical perinatal infection as well as a direct effect of the antibiotics on the fetal brain or cerebral blood flow. Alternatively the antibiotic might have negatively influenced microbial colonization of newborn children, with long-lasting consequences. There are some suggestions that antibiotics alter immune tolerance by changing the fetal gut flora, thus contributing to the substantial increase in the incidence of asthma, allergies, autoimmune diseases, autism, ADHD and other chronic conditions [73-75]. The main conclusion from all these interesting studies is that we should be very cautious in prescribing antibiotics to pregnant women in the absence of proven benefit (e.g. spontaneous preterm labor with intact membranes), while in situations clearly associated with increased risk of maternal, fetal and neonatal death (e.g. clinical signs of chorioamnionitis) antimicrobial therapy is necessary.

\section{Diagnosis and treatment of different clinical forms of urinary tract infection}

The criteria for diagnosis and treatment of UTI are more restrictive compared with the general population, since the potential risks concern not only an expectant mother but also her unborn child.

\section{Asymptomatic bacteriuria}

\section{Screening tests}

Given the evidence that effective antimicrobial therapy of ASB in pregnancy significantly reduces the risk of pyelonephritis and possibly also adverse fetal outcomes, routine screening for the presence of clinically significant bacteriuria in all pregnant women has become necessary. Urine culture remains the most reliable test allowing the diagnosis of ASB. According to recommendations developed by the IDSA (Infectious Diseases Society of America), significant bacteriuria in asymptomatic women is defined as bacterial monoculture in the quantity of $\geq 10^{5}$ colony-forming units (CFU) per $\mathrm{ml}$ in two consecutive mid-stream cleancatch urine specimens or $\geq 10^{2} \mathrm{CFU} / \mathrm{ml}$ in urine collected from single urinary bladder catheterization [76]. However, for practical and economic reasons the guidelines for routine screening in pregnancy accept a single urine culture taken between weeks 12 and 16, or at first prenatal visit (if later), although there is only an $80 \%$ probability that the woman has true bacteriuria (vs. 95\% with the original criteria) $[9,77]$. Due to this high rate of false positive results, in some centers women with a positive urine culture are asked to return within 1 week for the second testing, to avoid unnecessary treatment [11].

A question which remains unanswered is: should women in whom no ASB was detected upon the first examination have additional screening in later pregnancy? To date, repeated tests have been recommended only in high-risk women (with diabetes, sickle cell anemia, immunological defects, urinary tract abnormalities or a history of recurring infections before pregnancy) $[2,38]$. However, the more recent reports suggest that repeating the urine culture in each trimester improves the detection rate of $\operatorname{ASB}[78,79]$. Mclsaac et al. studied 1050 women who were subjected to successive urine cultures before week 20, at week 28 and at week 36 [78]. A total of 49 cases of ASB were detected (prevalence $4.7 \%$ ). The authors demonstrated that basing the diagnosis on a single urine culture before 20 weeks' gestation leaves more than one-half of ASB cases undiagnosed, since $40.8 \%$ of diagnoses were made after the first culture vs. $63.3 \%$ after the second vs. $87.8 \%$ after the third culture. In a much smaller Turkish study, ASB prevalence distribution in the first, second, and third trimesters was $0.9 \%$, $1.83 \%$, and $5.6 \%$, respectively [79]. That suggests that many women with no bacteriuria in their initial examination in the first trimester may develop bacteriuria during the later trimesters. The authors of these studies conclude that it would be prudent to screen pregnant women for bacteriuria also in the second and third trimesters [78, 79]. However, until large, prospective, randomized clinical trials (RCTs) are available and a clear benefit of this routine additional screening is observed, no recommendation can be made for or against it.

\section{Treatment}

The presence of ASB in a pregnant woman is an absolute indication for initiation of the treatment. The benefits of such a strategy with bacteriological follow-up were summarized by Smaill and Vazquez for the Cochrane Library, on the basis of the results of 14 RCTs, embracing 2302 pregnant women with ASB, in which the effects of different antibiotics given for different duration were compared to placebo or untreated groups [27]. The analysis of 5 of these trials, involving 
820 pregnancies, showed that antibiotics effectively cleared ASB ( $R R=0.25,95 \% \mathrm{Cl}: 0.14-0.48)$. In the same review the authors present the results of another analysis which included 11 trials and 1955 pregnancies, which demonstrated that antibiotic treatment of ASB may reduce the incidence of pyelonephritis by $52-86 \%(\mathrm{RR}=0.25,95 \% \mathrm{Cl}$ : 0.14-0.48).

Management of ASB in pregnancy consists of short-term, usually 5-7 days, oral antibiotic therapy [76]. Basic principles of management are presented in Table II. In the face of the rapidly developing antibiotic resistance, the current position is that the treatment should be based on microbial sensitivity testing. Recently, a growing number of authors suggest that a reasonable first choice drug in the second and third trimester is the old and almost forgotten nitrofurantoin [80-82]. As shown by most recent studies, nitrofurantoin is active against nearly $90 \%$ of $E$. coli strains isolated from urine, including $89 \%$ of extended spectrum $\beta$-lactamase (ESBL)-producing strains [81, 82]. Kashanian et al. carried out a retrospective analysis of drug resistance among bacteria cultured from urine specimens in a single hospital in New York in 2003-2007 [81]. Out of 10417 cultures in which E. coli growth was achieved, $95.6 \%$ were sensitive to nitrofurantoin, with the average resistance rate of $2.3 \%$, being significantly lower than that of ciprofloxacin, levofloxacin and trimethoprim/sulfamethoxazole (24.2\%, $24 \%$ and $29 \%$, respectively). A single $3 \mathrm{~g}$ dose of phosphomycin also has a low resistance rate in $E$. coli infections and seems to be effective in non-pregnant women, but there is limited experience in using this regimen in pregnancy, and until more data become available it should not be given [50].

Women with GBS isolated from the urine at any point during pregnancy should be treated according to the CDC (Centers for Disease Control and Prevention) guidelines, revised in 2010 and endorsed by the ACOG (American College of Obstetricians and Gynecologists) and AAP (American Academy of Pediatrics) [24]. In asymptomatic women with urinary colony counts $<100000$ CFU/ $\mathrm{ml}$, antimicrobial agents are not recommended before the intrapartum period, since such treatment is not effective in eliminating GBS carriage or preventing neonatal disease and can cause adverse consequence. Symptomatic UTI or GBS significant ASB should be treated according to current standards of pregnancy care $[25,77]$. All of them (regardless of level of CFU/ml), at the time of labor or rupture of membranes, should receive appropriate intravenous antibiotics for the prevention of early-onset neonatal GBS disease, and do not need rescreening by genital or urinary tract culture in the third trimester, as they are presumed to be GBS colonized [24, 83, 84]. The same approach is recommended for women who had a previous in fant with invasive GBS disease. All other patients should be screened at 35-37 weeks' gestation for vaginal and rectal GBS colonization [24,84].

\section{Follow-up urine cultures}

All pregnant women with ASB should have periodic screening after therapy, since as many as one third of them experience a recurrent infection [58, 76]. Follow-up cultures should be obtained 1-2 weeks after treatment and then repeated once a month $[58,76]$. In case of persistent or recurrent bacteriuria, longer antibiotic therapy using the same agent (e.g. 7 instead of 3 days of treatment) or another first line drug is recommended. Subsequent treatment courses are administered until the bacterial counts drop to non-significant levels [56]. If bacteriuria persists despite repeated courses of therapy, as well as in women with additional risk factors (e.g. immunosuppression, diabetes, sickle cell anemia, neurogenic bladder) or recurrent/persistent UTIs before pregnancy, one should consider antimicrobial prophylaxis

Table II. Diagnosis and treatment of asymptomatic bacteriuria (ASB) and acute cystitis/urethritis (doses for normal renal function)

\begin{tabular}{|lcc|}
\hline & Asymptomatic bacteriuria & $\begin{array}{c}\text { Acute cystitis/ } \\
\text { urethritis }\end{array}$ \\
\hline Screening (obligatory) & $1^{\text {st }}$ prenatal visit or $12-16 \mathrm{HBD}$ & \\
\hline First line treatment & Amoxicillin $500 \mathrm{mg}$ every $8-12 \mathrm{~h}-$ for $3-7$ days & For 7 days \\
\cline { 2 - 3 } & Cephalexin $500 \mathrm{mg}$ every $12 / 6 \mathrm{~h}-$ for $3-7$ days & For 7 days \\
\hline FDA cat. B & Amoxicillin/clavulanic acid $500 \mathrm{mg}$ every $12 \mathrm{~h}-$ for $3-7$ days & For 7 days \\
\cline { 2 - 3 } & Nitrofurantoin $100 \mathrm{mg}$ every $12 \mathrm{~h}-$ for $5-7$ days & For 7 days \\
\cline { 2 - 3 } & Cefuroxime $250 \mathrm{mg}$, every $12 \mathrm{~h}-$ for $3-7$ days & For 7 days \\
\hline FDA cat. C & Cefpodoxime $100 \mathrm{mg}$ every $12 \mathrm{~h}$ & For 7 days \\
\hline
\end{tabular}

*Treatment limited to the $2^{\text {nd }}$ and $3^{\text {rd }}$ trimester (except last 2 weeks); should not be used in the $1^{\text {st }}$ trimester if other first line agents may be administered. 
$[10,56]$. Patients with recurrences associated with sexual activity may be offered postcoital prophylaxis a single antibiotic dose (e.g. nitrofurantoin 50-100 mg p.o. or cephalexin 250-500 mg p.o.) postcoitally $[56,85]$. The remaining women may be given small doses of antibacterial agents (e.g. nitrofurantoin 50-100 $\mathrm{mg}$ in the evening) until the end of the pregnancy. In this group the follow-up urine culture is performed only at the beginning of the third trimester. In case of significant bacteriuria, prophylactic doses should be replaced by another course of antimicrobials, based on susceptibility testing [56].

\section{Cystitis/urethritis}

\section{Diagnosis}

The diagnosis is made on the basis of symptoms (cloudy urine, dysuria, frequency, urgency, abdominal or suprapubic pain) and the presence of even small bacterial colony counts $\left(\geq 10^{2}-\right.$ $\left.10^{3} \mathrm{CFU} / \mathrm{ml}\right)[56]$.

\section{Management}

In most cases of lower UTI, the treatment is similar to that used in ASB (Table II) and should be guided by antimicrobial susceptibility testing. The optimal duration of treatment is unknown, but longer courses (5-7 days) of the therapy are generally suggested $[12,55,58,86]$. Follow-up urine cultures are recommended 1-2 weeks after the treatment and then once a month. In women receiving chronic immunosuppression, management discussed in the section on ASB should be followed. In women with recurrent acute cystitis, antimicrobial urinary suppression based on daily use of a small dose of antibacterial drug during the symptom-free period is recommended or, in the case of an evident relationship of the disease with sexual activity, only after intercourse (e.g. nitrofurantoin 50-100 mg, cephalexin 250-500 mg) $[56,85]$.

\section{Acute pyelonephritis}

Acute pyelonephritis is most common in late pregnancy, with $80-90 \%$ of cases occurring in the second and third trimester [16, 17, 29, 38, 87]. It is usually a consequence of undiagnosed or inappropriately treated lower UTI, or a complication of $30-40 \%$ of cases of untreated ASB [8]. The overall incidence of pyelonephritis reaches up to $2 \%$ of all pregnancies (vs. $<1 \%$ in the general population $[2,8]$. Besides ASB, the other risk factors of acute pyelonephritis include: mother's age, nulliparity, sickle cell anemia, diabetes, nephrolithiasis, illicit drug use, history of pyelonephritis and maternal urinary tract defects $[2,8,24,58]$.

The clinical presentation is typical and includes lumbar pain, fever of $>38^{\circ} \mathrm{C}$, chills, nausea, vomiting and costo-vertebral angle tenderness, while symptoms of dysuria are less common. Nearly one in five of pregnant women with pyelonephritis has septicemia at diagnosis [3, 8, 17, 40]. Hill et al. among 32282 pregnant women, who had been admitted to their prenatal clinic during the 2-year study period, identified 440 cases of pyelonephritis [17]. Complications included: anemia (23\%), septicemia (17\%), transient renal dysfunction (2\%), and pulmonary insufficiency (7\%). Numbers of preterm births and small-for-gestational-age infants were not increased as compared with expected rates in this hospital.

\section{Management}

Basic principles of management are presented in Table III. According to the 2005 IDSA guidelines, all suspected cases of pyelonephritis should be hospitalized at least for the initial $48 \mathrm{~h}$ of treatment [76]. However, some authors believe that in carefully selected cases, when a definite diagnosis of pyelonephritis can be made and a strict medical follow-up is possible, outpatient treatment may be attempted $[38,88,89]$. This is the case mostly in young, hitherto healthy women before 24 weeks' gestation and free of severe symptoms

Table III. Diagnosis and treatment of acute pyelonephritis (doses for normal renal function)

\begin{tabular}{|ll|}
\hline Diagnosis & Symptoms + urine culture: \\
& Fever $>38^{\circ} \mathrm{C}$, lumbar pain, skeletal and joint pains, nausea/vomiting \\
& with or without accompanying dysuria, polyuria \\
& $\geq 10^{5} \mathrm{CFU} / \mathrm{ml}$ in mid-stream urine specimen \\
\hline Mild or moderate acute pyelonephritis & Ceftriaxone $1 \mathrm{~g}$ every $24 \mathrm{~h}$ \\
& Cefepime $1 \mathrm{~g}$ every $24 \mathrm{~h}$ \\
& Amoxicillin with clavulanic acid $1.2 \mathrm{~g}$ every $12 \mathrm{~h}$ \\
& Aztreonam $1 \mathrm{~g}$ every $8-12 \mathrm{~h}$ \\
\hline Severe acute pyelonephritis/immuno- & Ticarcillin with clavulanic acid $3.1 \mathrm{~g}$ every $6 \mathrm{~h}$ \\
suppression/urinary stasis & Piperacillin with tazobactam $3.375 \mathrm{~g}$ every $6 \mathrm{~h}$ \\
& Meropenem 0.5 g every $8 \mathrm{~h}$ \\
& Ertapenem $1 \mathrm{~g}$ every $24 \mathrm{~h}$ \\
& Doripenem $1 \mathrm{~g}$ every $8 \mathrm{~h}$ \\
\hline
\end{tabular}

In case of allergy to $\beta$-lactams. 
such as high fever with chills, persistent vomiting, significant dehydration or clinical signs of sepsis (tachycardia, tachypnea or hypotension) [38, 39, 49]. It should be stressed, however, that these are only opinions, based mostly on observational and a few small RCTs, and that in contrast to the overall population, evidence regarding the safety of such management is not available [89-91]. Appropriate hydration of the patient is a very important part of the treatment regardless of the setting. Beside urine and blood culture, recommendations include basic laboratory analyses (complete blood counts, electrolytes, creatinine, liver parameters, coagulation profile) and an ultrasound scan, which usually reveals dilation of pyelocalyceal systems and allows exclusion of other causes of the symptoms (e.g. renal abscess, ureter obstruction, other abdominal infections).

In all patients, regardless of whether they are hospitalized, antibiotics should be given parenterally, for at least the first $48 \mathrm{~h}$ (until the resolution of fever). Usually the treatment is initiated empirically and verified after obtaining the microbial sensitivity test results $[1,76]$. Forty-eight hours after resolution of symptoms, administration may be switched to the oral route. In case of fever persisting for more than $48 \mathrm{~h}$, blood and urine cultures should be repeated, and any possible causes of treatment failure (perirenal abscess, lithiasis, congenital or acquired structural changes within the urinary tract) have to be carefully considered $[38,76]$. Antibiotic therapy is usually continued for 10-14 days, although its optimum duration has never been established.

Unfortunately, there are not sufficient data available to recommend the specific treatment regimens in pregnant women. $\beta$-Lactam antibiotics are used most commonly, as they are relatively safe for the fetus (Table III). Carbapenems are reserved for the treatment of more severe cases, and those caused by multi-drug resistant bacteria. Administration of more toxic agents, such as aminoglycosides (potential fetal neuro- and nephrotoxicity) is acceptable only in cases when the expected benefits for the mother (e.g., in life-threatening conditions) outweigh the potential risk to the unborn child.

Recurrences of pyelonephritis, observed in 6-8\% of pregnant women, pose a significant problem. In such cases, in periods free of symptoms, prophylactic treatment is recommended (e.g. nitrofurantoin 50-100 mg, cephalexin 250-500 mg before sleep), with urine culture at the beginning of the third trimester. Then upon detection of bacteriuria, prophylaxis is replaced by regular treatment [56]. In the aforementioned prospective study by Hill et al., after the successful treatment of pyelonephritis all studied women were placed on uri- nary suppression with nitrofurantoin, $100 \mathrm{mg}$ daily, and were carefully followed up [17]. Only 12 of them $(2.7 \%)$ were readmitted for recurrent pyelonephritis, and all 12 were found to be noncompliant with their antimicrobial suppression. However, again, this regimen is not supported by evidence obtained in RCTs. Recent analysis of the results of a randomized study that included 200 pregnant women showed no superiority of nitrofurantoin prophylaxis combined with standard of care (careful bacteriological control and antibiotic therapy upon detection of bacteriuria) over the standard of care alone [92].

\section{Future challenges}

Due to the potential risk to mother and fetus, detection and effective treatment of UTIs remains an important clinical problem. It is advisable to assess risk factors for UTI in pregnancy, bearing in mind that some diagnostic procedures are not feasible and advisable to perform i.e. urodynamic studies [93]. Unfortunately, in contrast to the overall population, available data are scant, and the management guidelines were published several years ago and were largely opinion-based. The development of new recommendations requires well-planned, extensive studies, that would answer the still open questions regarding the frequency of screening and follow-up examinations, purposefulness of prophylaxis, safety of hitherto insufficiently studied or new antibiotics in pregnancy, and choice of optimum treatment regimens. If possible, any antibiotic use should be avoided in the first trimester, as this is the period of fetal organogenesis and nervous system development, with the highest risk of teratogenic effects of drugs.

Another disturbing problem, particularly in the aspect of fetal safety associated with therapeutic limitations, is the observed rapid development of antibiotic resistance. In general, this is applicable to diverse bacterial pathogens in many different clinical settings, and is becoming one of the most significant future threats to public health. In Gram-negative bacilli the resistance is associated with their ability to synthesize extended spectrum $\beta$-lactamases (ESBLs), as well as carbapenemases. The rapid spread of resistance is due to the fact that genes encoding $\beta$-lactamases and carbapenemases (particularly of the KPC type) are localized on mobile genomic elements (plasmids) easily transferable within the strain and among different strains of bacteria, even if the bacteria are not related to each other [94]. The introduction of new diagnostic methods with genetic typing may provide new opportunities in this area [95].

It is believed that currently more than a half of E. coli strains and more than one third of Klebsiella 
are $\mathrm{ESBL+}$, leading to the resistance to third generation cephalosporins $[15,78,80]$. Enterobacteriaceae strains are resistant to all $\beta$-lactam and carbapenem antibiotics [94]. Another commonly observed phenomenon that has been known already for some years is meticillin resistance of Gram-positive cocci, which in practice often translates to multidrug resistance of these bacteria. One should also remember that antibiotic resistance of bacteria may differ depending on geographic area, hospital and even hospital ward, and the information on this topic may be crucial when making therapeutic decisions. Despite the diet in pregnancy is not generally different [96], we may think about some dietary approaches to change urinary $\mathrm{pH}$ as a prophylaxis of UTI in pregnancy.

\section{Conflict of interest}

The authors declare no conflict of interest.

\section{References}

1. Schnarr J, Smaill F. Asymptomatic bacteriuria and symptomatic urinary tract infection in pregnancy. Eur J Clin Invest 2008; 38 (Suppl. 2): 50-7.

2. Farkash E, Wientraub AY, Sergienko R, et al. Acute antepartum pyelonephritis in pregnancy: a critical analysis of risk factors and outcomes. Eur J Obstet Gynecol Reprod Biol 2012; 162: 24-7.

3. Gravett MG, Martin ET, Bernson JD, et al. Serious and life-threatening pregnancy-related infections: opportunities to reduce the global burden. Plos Med 2012; 9: e1001324.

4. Foxman B. Epidemiology of urinary tract infections: incidence, morbidity, and economic costs. Am J Med 2002; 113: 5-13.

5. Mazor-Dray E, Levy A, Schlaeffer F, Sheiner E. Maternal urinary tract infection: is it independently associated with adverse pregnancy outcome? J Matern Fetal Neonatal Med 2009; 22: 124-32.

6. Bolton M, Horvath DJ, Li B, et al. Intrauterine growth restriction is a direct consequence of localized maternal uropathogenic Escherichia coli cystitis. Plos ONE 2012; 7: 1-9.

7. Jeyabalan A, Lain KY. Anatomic and functional changes of the upper urinary tract during pregnancy. Urol Clin North Am 2007; 34: 1-6.

8. Jolley JA, Wing DA. Pyelonephritis in pregnancy: an update on treatment options for optimal outcomes. Drugs 2010; 70: 1643-55.

9. Lumbiganon P, Laopaiboon M, Thinkhamrop J. Screening and treating asymptomatic bacteriuria in pregnancy. Curr Opin Obstet Gynecol 2010; 22: 95-9.

10. Dwyer PL, O'Reilly M. Recurrent urinary tract infection in the female. Curr Opin Obstet Gynecol 2002; 14: 537-43.

11. MacLean $A B$. Urinary tract infection in pregnancy. Int J Antimicrob Agents 2001; 17: 273-7.

12. Duarte G, Marcolin AC, Quintana SM, Cavalli RC. Urinary tract infection in pregnancy. Rev Bras Ginecol Obstet 2008; 30: 93-100.

13. BahadiA, El Kabbaj D, Elfazazi $H$, et al. Urinary tract infection in pregnancy. Saudi J Kidney Dis Transpl 2010; 21: 342-4.
14. Wagenlehner FM, Weidner W, Naber KG. An update on uncomplicated urinary tract infections in women. Curr Opin Urol 2009; 19: 368-74.

15. Sabharwal ER. Antibiotic susceptibility patterns of uropathogens in obstetric patients. N Am J Med Sci 2012; 4: 316-9.

16. Sharma P, Thapa L. Acute pyelonephritis in pregnancy: a retrospective study. Aust N Z J Obstet Gynaecol 2007; 47: 313-5.

17. Hill JB, Sheffield JS, McIntire DD, Wendel GD Jr. Acute pyelonephritis in pregnancy. Obstet Gynecol 2005; 105: 18-23.

18. Stenqvist K, Dahlén-Nilsson I, Lidin-Janson G, et al. Bacteriuria in pregnancy: frequency and risk of acquisition. Am J Epidemiol 1989; 129: 372-9.

19. Pastore LM, Savitz DA, Thorp JM Jr. Predictors of urinary tract infection at the first prenatal visit. Epidemiology 1999; 10: 282-7.

20. Pastore LM, Savitz DA, Thorp JM Jr, et al. Predictors of symptomatic urinary tract infection after 20 weeks' gestation. J Perinatol 1999; 19: 488-93.

21. Kovavisarach E, Vichaipruck M, Kanjarahareutai S. Risk factors related to asymptomatic bacteriuria in pregnant women. J Med Assoc Thai 2009; 92: 606-10.

22. Turck M, Goffe BS, Petersdorf RG. Bacteriuria of pregnancy. Relation to socioeconomic factors. N Engl J Med 1962; 266: 857-60.

23. Thurman AR, Steed LL, Hulsey T, Soper DE. Bacteriuria in pregnant women with sickle cell trait. Am J Obstet Gynecol 2006; 194: 1366-70.

24. Verani JR, McGee L, Schrag SJ. Prevention of perinatal group B streptococcal disease revised guidelines from CDC, 2010. MMWR 2010; 59: 1-31.

25. McKenna DS, Matson S, Northern I. Maternal group B streptococcal (GBS) genital tract colonization at term in women who have asymptomatic GBS bacteriuria. Infect Dis Obstet Gynecol 2003; 11: 203-7.

26. Schrag SJ, Phil D, Zywicki S, et al. Group B streptococcal disease in the era of intrapartum antibiotic prophylaxis. N Engl J Med 2000; 342: 15-20.

27. Smaill F, Vazquez JC. Antibiotics for asymptomatic bacteriuria in pregnancy. Cochrane Database Syst Rev 2007; 18: CD000490.

28. Delzell, Schieve LA, Handler A, et al. Urinary tract infection during pregnancy: its association with maternal morbidity and perinatal outcome. Am J Public Health 1994; 84: 405-10.

29. Mittendorf R, Williams MA, Kass EH. Prevention of preterm delivery and low birth weight associated with asymptomatic bacteriuria. Clin Infect Dis 1992; 14: 927-32.

30. Sheiner E, Mazor E, Levy A. Asymptomatic bacteriuria during pregnancy. J Matern Fetal Neonat Med 2009; 22: 423-7.

31. Romero R, Oyarzun E, Mazor M, et al. Metaanalysis of the relationship between asymptomatic bacteriuria and preterm delivery/low birth weight. Obstet Gynecol 1989; 73: 576-82.

32. Chen YK, Chen SF, Li HC, Lin HC. No increased risk of adverse pregnancy outcomes in women with urinary tract infections: a nationwide population-based study. Acta Obstet Gynecol Scand 2010; 89: 882-8.

33. Meis PJ, Michielutte R, Peters TJ, et al. Factors associated with preterm birth in Cardiff, Wales. I. Univariable and multivariable analysis. Am J Obstet Gynecol 1995; 173 : 590-6.

34. Meis PJ, Michielutte R, Peters TJ, et al. Factors associated with preterm birth in Cardiff, Wales. II. Indicated and 
spontaneous preterm birth. Am J Obstet Gynecol 1995; 173: $597-602$

35. Thomsen AC, Morup L, Brogaard Hansen K. Antibiotic elimination of group-B streptococci in urine in prevention of preterm labour. Lancet 1987; 1: 591-3.

36. Adair CE, Kowalsky L, Quon H, et al. Risk factors for early-onset group $\mathrm{B}$ streptococcal disease in neonates: a population based case-control study. CMAJ 2003; 169 198-203.

37. Oddie S, Embleton ND; the Northern Neonatal Network. Risk factors for early onset neonatal group B streptococcal sepsis: case-control study. BMJ 2002; 325: 308-11.

38. Adis Data Information. Standard treatment of pyelonephritis in pregnancy involves parenteral antibacterials and intravenous hydration. Drugs Ther Perspect 2011; 27: 13-7.

39. Millar LK, Cox SM. Urinary tract infections complicating pregnancy. Infect Dis Clin North Am 1997; 11: 13-26.

40. Cunningham FG, Lucas MJ. Urinary tract infections complicating pregnancy. Baillieres Clin Obstet Gynaecol 1994; 8: 353-73.

41. Cox SM, Shelbourne P, Mason R, et al. Mechanisms of hemolysis and anemia associated with acute antepartum pyelonephritis. Am J Obstet Gynecol 1991; 164 587-90.

42. Galajdova L. Pulmonary dysfunction in acute antepartum pyelonephritis and other pregnancy infections. J Obstet Gynecol 2010; 30: 654-8.

43. Conde-Agudelo A, Villar J, Lindheimer M. Maternal infection and risk of preeclampsia: systematic review and metaanalysis. Am J Obstet Gynecol 2008; 198: 7-22.

44. Mittal P, Wing DA. Urinary tract infections in pregnancy. Clin Perinatol 2005; 32: 749-64.

45. Neal DE Jr. Complicated urinary tract infections. Urol Clin North Am 2008; 35: 13-22.

46. Gravett MG, Rubens CE, Nunes TM; GAPPS Review Group. Global report on preterm birth and stillbirth (2 of 7): discovery science. BMC Pregnancy Childbirth 2010 10 (Suppl. 1): S2: 1-26.

47. Millar LK, DeBuque L, Wing DA. Uterine contraction frequency during treatment of pyelonephritis in preg nancy and subsequent risk of preterm birth. J Perinatal Med 2003; 31: 41-6.

48. Beck S, Wojdyla D, Say L, et al. The worldwide incidence of preterm birth: a systematic review of maternal mortality and morbidity. Bull World Health Organ 2010; 88: 31-8.

49. Torres M, Moayedi S. Gynecologic and other infections in pregnancy. Emerg Med Clin N Am 2012; 30: 869-84.

50. Widmer ZM, Gülmezoglu AM, Mignini L, Roganti A Duration of treatment for asymptomatic bacteriuria during pregnancy. Cochrane Database Syst Rev 2011; 7: CD000491.

51. Crider KS, Cleves MA, Reefhuis J, et al. Antibacterial medication use during pregnancy and risk of birth defects. National Birth Defects Prevention Study. Arch Pediatr Adolesc Med 2009; 163: 978-85.

52. Sulfonamides, nitrofurantoin, and risk of birth defects. Committee Opinion No. 494. American College of Obstetricians and Gynecologists. Obstet Gynecol 2011; 117: 1484-5.

53. Nordeng H, Lupattelli A, Romøren M, Koren G. Neonatal outcomes after gestational exposure to nitrofurantoin. Obstet Gynecol 2013; 121: 306-13.

54. Duff P. Antibiotic selection in obstetrics; making cost effective choices. Clin Obstet Gynecol 2002; 45: 59-72.

55. Guinto VT, De Guia B, Festin MR, Dowswell T. Different antibiotic regimens for treating asymptomatic bacteri- uria in pregnancy. Cochrane Database Syst Rev 2010; CD007855.

56. Hooton TM. Urinary tract infections and asymptomatic bacteriuria in pregnancy. UpToDate.com; updated 2011.

57. Forna F, McConnell M, Kitabire FN, et al. Systematic review of the safety of trimethoprim/sulfamethoxazole for prophylaxis in HIV-infected pregnant women: implications for resource-limited settings. AIDS Reviews 2006; 8: 24-36.

58. Berard A, Santos F, Ferreira E, Perreault S. Urinary tract infections during pregnancy. 2011; 113-32. www.intechopen.com.

59. Nuchprayoon I, Sanpavat S, Nuchprayoon S. Glucose-6-phosphate dehydrogenase (G-6-PD) mutation in Thailand: G6PD Viangchan (871GA) is the most common deficiency variant in the Thai population. Hum Mutat 2002; 19: 185-9.

60. Loebstein R, Addis A, Ho E, et al. Pregnancy outcome following gestational exposure to fluoroquinolones: a multicenter prospective controlled study. Antimicrob Agents Chemother 1998; 42: 1336-9.

61. Larsen H, Nielsen GL, Schønheyder HC, et al. Birth outcome following maternal use of fluoroquinolones. In J Antimicrob Agents 2001; 18: 259-62.

62. Santos F, Oraichi D, Berard A. Prevalence and predictors of anti-infective use during pregnancy. Pharmacoepidemiol Drug Saf 2010; 19: 418-27.

63. Lee M, Bozzo P, Einarson A, Koren G. Urinary tract infections in pregnancy. Can Fam Physician 2008; 54: 853-4

64. Bar-Oz B, Moretti ME, Boskovic R, O’Brien L, Koren. The safety of quinolones - a meta-analysis of pregnancy outcomes. Eur J Obstet Gynecol Reprod Biol 2009; 143: 75-8.

65. Dashe JS, Gilstrap LC. Antibiotic use in pregnancy. Obstet Gynecol Clin North Am 1999; 24: 617-29.

66. Macejko AM, Schaeffer AJ. Asymptomatic bacteriuria and symptomatic urinary tract infections during pregnancy. Urol Clin North Am 2007; 34: 35-42.

67. Källén BA, Otterblad Olausson P. Maternal drug use in early pregnancy and infant cardiovascular defect. Reprod Toxicol 2003; 17: 255-6.

68. Einarson A, Phillips E, Mawji F, et al. A prospective controlled multicentre study of clarithromycin in pregnancy. Am J Perinatol 1998; 15: 523-5.

69. Czeizel AE, Rockenbauer M, Sorensen HT, Olsen J. A population-based case-control teratologic study of oral erythromycin treatment during pregnancy. Reprod Toxicol 1999; 13: 531-6.

70. Bar-Oz, Weber-Schoendorfer C, Barlin M, et al. The outcomes of pregnancy in women exposed to the new macrolides in the first trimester. A prospective, multicentre, observational study. Drug Saf 2012; 35: 589-98.

71. Lin KJ, Mitchell AA, Yau WP, et al. Safety of macrolides during pregnancy. Am J Obstet Gynecol 2013; 208: 221. e1-8.

72. Kenyon S, Pike K, Jones DR, et al. Childhood outcomes after prescription of antibiotics to pregnant women with spontaneous preterm labour: 7-year follow-up of the ORACLE II trial. Lancet 2008; 372: 1319-27.

73. McKeever TM, Lewis SA, Smith C, et al. The importance of prenatal exposures on the development of allergic disease: a birth cohort study using the West Midlands General Practice Database. Am J Respir Crit Care Med 2002; 166: 827-32.

74. Bedford Russel AR, Murch SH. Could peripartum antibiotics have delayed health consequences for the infant? BJOG 2006; 113: 758-65. 
75. Algert CS, Bowen JR, Lain SL, et al. Pregnancy exposures and risk of childhood asthma admission in a population birth cohort. Pediatr Allergy Immunol 2011; 22: 836-42.

76. Nicolle LE, Bradley S, Colgan R, et al. Infectious Diseases Society of America guidelines for the diagnosis and treatment of asymptomatic bacteriuria in adults. Clin Infect Dis 2005; 40: 643-54.

77. Lin K, Fajaro K, U.S. Preventive Services Task Force. Screening for asymptomatic bacteriuria in adults: evidence for the U.S. Preventive Services Task Force reaffirmation recommendation statement. Ann Intern Med 2008; 149: W20-24, 43-7.

78. Mclsaac W, Carrol JC, Biringer A, et al. Screening for as ymptomatic bacteriuria in pregnancy. J Obstet Gynaecol Can 2005; 27: 20-4.

79. Tugrul S, Oral O, Kumru P, et al. Evaluation and importance of asymptomatic bacteriuria in pregnancy. Clin Exp Obstet Gynecol 2005; 32: 237-40.

80. Rizvi M, Khan F, Shukla, et al. Rising prevalence of antimicrobial resistance in urinary tract infections during pregnancy: necessity for exploring newer treatment options. J Lab Physicians 2011; 3: 98-103.

81. Kashanian J, Hakimian P, Blute M Jr, et al. Nitrofurantoin: the return of an old friend in the wake of growing resistance. BJU Int 2008; 102: 1634-7.

82. AshaPai KB, Rekha R, Sanjeev $H$, et al. Nitrofurantoin: an alternative therapy for uncomplicated cystits in the era of antimicrobial resistance. J Clin Diagn Res 2011; 5: 964-6.

83. Allen VM, Yudin MH, Bouchard C, et al.; Infectious Diseases Committee, Society of Obstetricians and Gynaecologists of Canada. Management of group B streptococcal bacteriuria in pregnancy. J Obstet Gynaecol Can 2012; 34: 482-6.

84. American College of Obstetricians and Gynecologists Committee on Obstetric Practice. ACOG Committee Opinion No. 485. Obstet Gynecol 2011; 117: 1019-28.

85. Harris RE, Gilstrap LC 3rd. Cystitis during pregnancy: a distinct clinical entity. Obstet Gynecol 1981; 57: 578-80.

86. Gilstrap LC 3rd, Ramin SM. Urinary tract infections during pregnancy. Obstet Gynecol Clin North Am 2001; 28: 581-91.

87. Archabald KL, Friedman A, Raker CA, Anderson BL. Impact of trimester on morbidity of acute pyelonephritis in pregnancy. Am J Obstet Gynecol 2009; 201: 406e1-4.

88. Lawrence I. Outpatient treatment evolves for pyelonephritis in pregnancy. Emerg Med News 2002: 24: 6.

89. Brooks AM, Garite TJ. Clinical trial of the outpatient management of pyelonephritis in pregnancy. Infect Dis Obstet Gynecol 1995; 3: 50-5.

90. Wing DA, Hendershott CM, Debuque L, Millar LK. Outpatient treatment of acute pyelonephritis in pregnancy after 24 weeks. Obstet Gynecol 1999; 94: 683-4.

91. Millar LK, Wing DA, Paul RH, Grimes DA. Outpatient treatment of pyelonephritis in pregnancy: a randomized controlled trial. Obstet Gynecol 1995; 86: 560-4.

92. Schneeberger C, Geerling SE, Middleton P, Crowther CA. Interventions for preventing recurrent urinary tract infection during pregnancy. Cochrane Database Syst Rev 2012; 11: CD009279.

93. Dybowski B, Bres-Niewada E, Radziszewski P. Pressure-flow nomogram for women with lower urinary tract symptoms. Arch Med Sci 2014; 10: 752-6.

94. Carlet J, Jarlier V, Harbath, et al. Ready for a world without antibiotics? The Pensières antibiotic resistance call to action. Antimicrob Resist Infect Control 2012; 1: 1-23.

95. Sękowska A, Gospodarek E, Kamińska D. Antimicrobial susceptibility and genetic similarity of ESBL-positive
Klebsiella pneumoniae strains. Arch Med Sci 2012; 8: 993-7.

96. Bojar I, Owoc A, Humeniuk E, Fronczak A, Walecka I. Quality of pregnant women's diet in Poland - macro-elements. Arch Med Sci 2014; 10: 361-5. 